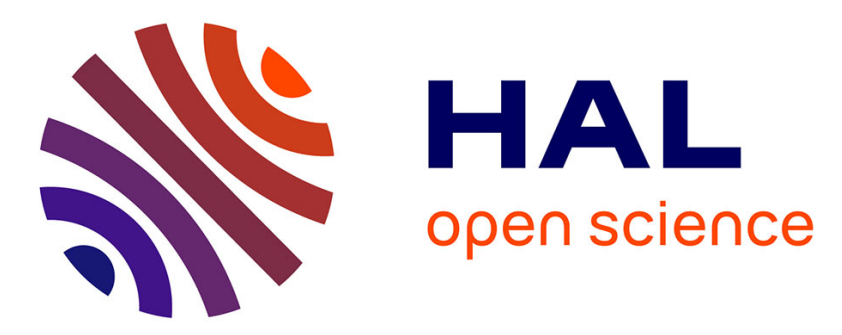

\title{
A Hybrid Bulk-Bin Approach to Model Warm-Rain Processes
}

Wojciech Grabowski, Odile Thouron, Jean-Pierre Pinty, Jean-Louis Brenguier

\section{To cite this version:}

Wojciech Grabowski, Odile Thouron, Jean-Pierre Pinty, Jean-Louis Brenguier. A Hybrid Bulk-Bin Approach to Model Warm-Rain Processes. Journal of the Atmospheric Sciences, 2010, 67 (2), pp.385399. 10.1175/2009JAS3155.1. hal-00993290

\section{HAL Id: hal-00993290 \\ https://hal.science/hal-00993290}

Submitted on 8 Feb 2022

HAL is a multi-disciplinary open access archive for the deposit and dissemination of scientific research documents, whether they are published or not. The documents may come from teaching and research institutions in France or abroad, or from public or private research centers.
L'archive ouverte pluridisciplinaire HAL, est destinée au dépôt et à la diffusion de documents scientifiques de niveau recherche, publiés ou non, émanant des établissements d'enseignement et de recherche français ou étrangers, des laboratoires publics ou privés. 


\title{
A Hybrid Bulk-Bin Approach to Model Warm-Rain Processes
}

\author{
WOJCIECH W. GRABOWSKI \\ National Center for Atmospheric Research, * Boulder, Colorado \\ ODILE THOURON \\ CNRM/GAME Météo-France/CNRS, Toulouse, France \\ JEAN-PIERRE PINTY \\ Laboratoire d'Aérologie, University of Toulouse, and CNRS, Toulouse, France \\ JEAN-LOUIS BRENGUIER \\ CNRM/GAME Météo-France/CNRS, Toulouse, France
}

\begin{abstract}
This paper presents a hybrid approach to model warm-rain processes, merging the diverse schemes of bulk and detailed (bin) microphysics. In the bulk scheme, the key assumption is that the exact saturation is maintained inside a cloud. In contrast, the supersaturation inside a cloud is predicted in the bin scheme and is applied to calculate the diffusional growth of cloud droplets. Predicting the supersaturation is numerically cumbersome, however, and typically requires spatial and temporal resolutions that are significantly higher than those that can be applied in the bulk scheme. At the same time, supersaturations inside clouds are small, and the condensate amounts in bulk and bin schemes differ insignificantly. This critical observation forms a starting point for the hybrid bulk-bin approach. In this approach, when the cloud water first appears, the activation scheme inserts cloud droplets at the low end of the bin representation. Subsequent diffusional and eventually accretional growth shift the spectrum toward larger sizes so that the saturation inside a cloud is maintained. Details of the hybrid approach are discussed in this paper, and the validation against the traditional bin scheme in a framework of the adiabatic rising parcel is presented.

Before the scheme can be applied to the multidimensional cloud model, a 1D advection-condensation problem of Grabowski and Smolarkiewicz is used to address the issue of the numerical difficulties that finitedifference schemes experience near cloud edges. In the bulk case, these are in the form of condensation rate overshoots and undershoots; and this aspect requires special attention in the hybrid scheme. A novel approach is developed that provides a physically consistent solution near cloud edges using the hybrid bulk-bin scheme. The key is to allow grid boxes near the edges to be partly cloudy and to include spectral changes of cloud droplets that take this into account. Application of the hybrid scheme to an idealized 2D problem of moist thermal rising from rest and producing rain illustrates the application of the scheme to practical problems of cloud dynamics and warm-rain microphysics.
\end{abstract}

* The National Center for Atmospheric Research is sponsored by the National Science Foundation.

Corresponding author address: Wojciech W. Grabowski, NCAR/ MMM, P.O. Box 3000, Boulder, CO 80307-3000.

E-mail: grabow@ncar.ucar.edu

\section{Introduction}

Modeling microphysical processes in warm (ice-free) clouds involves representation of cloud droplet activation and their growth by the diffusion of water vapor and by collision-coalescence. These are commonly referred to as warm-rain processes. Approaches with various degrees of 
complexity have been developed over last several decades to represent warm-rain processes in numerical models of clouds. In the continuous medium representation, the most comprehensive approach (commonly referred to as the detailed or bin microphysics) represents the spectrum of cloud droplets and drizzle/rain drops applying a finite number of size classes (e.g., Clark 1973; Feingold et al. 1988, 1994; Kogan 1991; Ackerman et al. 2004). However, such an approach is computationally demanding. First, because the range of drop sizes is large (from about $1 \mu \mathrm{m}$ to about $1 \mathrm{~cm}$ ), at least a few dozens of size (or mass) classes (bins) need to be considered to faithfully represent evolution of the spectrum. In a multidimensional cloud model, advection of all classes in the physical space requires significant computational effort. Second, prediction of the spectral changes due to diffusion of water vapor requires prediction of the supersaturation field that ultimately determines the rate of diffusional growth or evaporation of cloud droplets. When model vertical resolution is coarse, prediction of the supersaturation is cumbersome, especially near the cloud base (e.g., Clark 1974; Morrison and Grabowski 2008) and near cloud edges (see Grabowski and Morrison 2008 for a discussion and a list of relevant references). Finally, spectral changes due to collision-coalescence need to be modeled using by the Smoluchowski equation (Smoluchowski 1916), typically referred to as the stochastic coalescence equation in cloud physics literature (e.g., Pruppacher and Klett 1997), and accurate solution of this equation is not trivial.

As long as only gross characteristics of the condensed water are of interest, one can dramatically simplify the numerical treatment of condensational and accretional growth of cloud droplets and drizzle/rain drops by using a bulk approach. The bulk microphysics scheme splits the condensed water into two separate categories: the typically nonsedimenting cloud droplets and drizzle/rain drops with nonvanishing sedimentation velocity. The key assumption is that water saturation is maintained inside a cloud through instantaneous adjustment of the cloud water content. ${ }^{1}$ Saturation adjustment provides a simple and computationally efficient way to derive the condensation rate. The bulk scheme assumes that cloud droplets and drizzle/rain drops follow prescribed size distributions and only predicts the evolution of the distribution parameters. In its simplest incarnation proposed by Kessler (1969), the bulk scheme applies only

\footnotetext{
${ }^{1}$ Note that this assumption can be questioned for the turbulent cloud-environment mixing given the typical grid length of highresolution cloud models (tens of meters) and the length scale at which microscopic homogenization takes place (typically smaller than $1 \mathrm{~cm}$ ); see discussions in Grabowski 2007 and Jarecka et al. 2009.
}

two model variables, the cloud water and the drizzle/ rainwater mixing ratios, with the drizzle/rain drops assumed to follow the Marshall-Palmer size distribution. More sophisticated schemes predict either two or three distribution parameters (e.g., the number concentration, mixing ratio, shape parameter, etc.) and are referred to as double- or triple-moment bulk schemes (e.g., Meyers et al. 1997; Khairoutdinov and Kogan 2000; Seifert and Beheng 2001; Milbrandt and Yau 2005; Morrison et al. 2005). A bulk scheme can also predict the supersaturation, which provides a computationally efficient alternative to the bin scheme because the number of model variables is dramatically reduced (e.g., Morrison and Grabowski 2007, 2008).

This paper presents yet another alternative. Since supersaturations inside ice-free clouds are small, typically smaller than $1 \%$, one can apply the condensation rate derived using the saturation adjustment to predict corresponding evolution of the spectrum of cloud droplets and drizzle/rain drops. For the case of diffusional growth alone, this idea has already been applied in the $b^{2}$ scheme of Brenguier and Grabowski (1993). Here, we expand this approach to the case of precipitating clouds. Since this approach incorporates elements of both bulk and bin schemes (saturation adjustment and spectral representation, respectively), we refer to the new approach as the hybrid bulk-bin scheme.

As will become clear when details of the new approach are presented, the hybrid scheme does not predict activation of cloud droplets and has to be combined with the activation parameterization. It follows that the scheme is best suited for models that apply relatively coarse vertical resolution (vertical grid length of several tens of meters and larger), such as most large-eddy simulation models and all cloud-resolving models. As discussed in Clark (1974) and more recently in Morrison and Grabowski (2008, section $4 a)$, the vertical model grid length has to be as small as a few meters for low vertical velocities to accurately simulate cloud droplet activation-that is, to capture the maximum supersaturation near the cloud base that determines the total number of activated droplets. Such high spatial resolution is seldom afforded in cloud simulations and thus the approach proposed here provides a valuable alternative. The approach also addresses the issue of the numerical artifacts that highresolution models face near cloud edges. These artifacts impact the predicted supersaturation field and relevant microphysical characteristics as first noted in Grabowski (1989) and subsequently discussed in Stevens et al. (1996) and Grabowski and Morrison (2008).

The paper is organized as follows. The next section presents the theoretical formulation of the hybrid bulk-bin scheme. In section 3, we compare results from the hybrid 
scheme and the traditional bin microphysics scheme using the framework of the rising adiabatic parcel. Section 4 discusses application of the hybrid scheme to the 1D advection-condensation problem of Grabowski and Smolarkiewicz (1990, hereafter GS90) This simple test was used in the past to highlight numerical problems that finite-difference schemes face near cloud boundaries (e.g., Stevens et al. 1996; Grabowski and Morrison 2008). The test provides an excellent test bed before the hybrid scheme is used in realistic multidimensional cloud simulations. An example of such a simulation using a highly idealized 2D rising thermal setup is presented in section 5 . A brief summary in section 6 concludes the paper.

\section{Formulation of the bulk-bin scheme}

The equations describing the hybrid bulk-bin scheme merge representations of warm-rain processes applied in bulk and bin schemes. They consist of conservation equations of the potential temperature $\theta$, the water vapor mixing ratio $q_{v}$, and the condensed liquid water. For the latter, the spectral density function $f(r)$ is applied to represent the spectrum of cloud droplets and drizzle/rain drops, with $f(r) \equiv d n(r) / d r$, where $n(r)$ is the concentration, per unit mass of dry air, of drops smaller than $r$ (i.e., the cumulative number concentration). For the anelastic system, these equations can be written as

$$
\begin{gathered}
\frac{\partial \theta}{\partial t}+\frac{1}{\rho_{o}} \nabla \cdot\left(\rho_{o} \mathbf{u} \theta\right)=\frac{L_{v}}{\Pi c_{p}}(C-E), \\
\frac{\partial q_{v}}{\partial t}+\frac{1}{\rho_{o}} \nabla \cdot\left(\rho_{o} \mathbf{u} q_{v}\right)=-C+E, \\
\frac{\partial f}{\partial t}+\frac{1}{\rho_{o}} \nabla \cdot\left\{\rho_{o}\left[\mathbf{u}-\mathbf{k} v_{t}(r)\right] f\right\}+\frac{\partial}{\partial r}\left(\frac{d r}{d t} f\right) \\
=\left(\frac{\partial f}{\partial t}\right)_{\text {act }}+\left(\frac{\partial f}{\partial t}\right)_{\text {coal }},
\end{gathered}
$$

where $\mathbf{u}$ is the fluid flow velocity and $v_{t}(r)$ is the sedimentation velocity of droplets and drops ( $\mathbf{k}$ is the unit vector in the vertical direction); $\rho_{o}(z)$ is the anelastic base state density profile; $\Pi=\left(p / p_{o}\right)^{R / c_{p}}$ is the Exner function ( $p$ is the ambient pressure profile and $p_{o}=$ $1000 \mathrm{hPa}) ; L_{v}$ and $c_{p}$ are the latent heat of condensation and specific heat at constant pressure, respectively; $C$ is the condensation rate, that is, the diffusional growth/ evaporation of cloud droplets; and $E$ is the evaporation rate of drizzle/rain drops in subsaturated conditions. In (1c), the third term on the left-hand side represents growth of cloud droplets by the condensation of water vapor [term $C$ in (1a) and (1b)], as well as the evaporation of drizzle/rain drops in subsaturated air [term $E$ in (1a) and (1b)]. These processes are represented by the advection of $f$ in the radius space, with $d r / d t$ being the rate of change of the droplet/drop radius $r$ due to condensation or evaporation. The two terms of the rhs of (1c) represent the cloud droplet activation (i.e., the initial source of cloud droplets) and changes of the spectral density function due to collision-coalescence. Note that we exclude subgrid-scale turbulent diffusion terms in theoretical developments and computational examples presented in this paper.

In the bin scheme, the condensation and evaporation rates $C$ and $E$ are calculated from the predicted evolution of the spectral density function. For that, one needs to predict the supersaturation $S$ because the rate of change of the drop radius $r$ due to condensation/evaporation is given by $d r / d t=f_{\text {vent }} A S / r$, where $f_{\text {vent }}$ is the ventilation coefficient (i.e., the enhancement factor of the condensational growth rate or evaporation for a drop falling at terminal velocity in comparison to the motionless drop) and $A \approx 10^{-10} \mathrm{~m}^{2} \mathrm{~s}^{-1}$. The ventilation effects, negligible for condensation/evaporation of cloud droplets but important for evaporation of drizzle/rain drops, can be included in a standard way [cf. (13.60) and (13.61) in Pruppacher and Klett 1997]. Note that we neglect Kelvin and Raoult effects as well as gas kinetic corrections. Accurate prediction of the supersaturation typically requires high spatial and temporal resolution, especially near the cloud base when activation of cloud droplets takes place. In contrast, predicting the condensation/ evaporation rate of cloud water through the saturation adjustment imposes more relaxed constrains. In a nutshell, the hybrid bulk-bin approach predicts the evolution of the droplet spectra given the saturation-maintaining condensation rate.

An important aspect of (1) is the separation of the condensation rate of cloud droplets $C$ and the evaporation rate of drizzle/rain drops $E$. This is required because when saturation adjustment dictates evaporation, only cloud droplets can be assumed to respond instantaneously and drizzle/rain drops have to evaporate on a finite time scale, consistent with much smaller total surface area to volume ratio of drizzle/rain drops compared to cloud droplets. Note that for the case of evaporation, $C$ and $E$ are typically mutually exclusive: if $C<0$ but there is still some cloud water left after adjustment, then $E=0$ (i.e., a cloudy gridpoint); if $C=0$, then $E$ can be nonzero (a subsaturated cloud-free gridpoint). In the latter case, the drizzle/rain evaporation rate $E$ can be calculated as a shift of the drizzle/rain part of the spectrum given the negative supersaturation $S$. It then follows that to calculate saturation-maintaining condensation rate in the hybrid scheme, the spectrum of cloud and drizzle/rain drops has to be divided into two 
parts corresponding to the cloud water and the drizzle/ rain. The threshold droplet radius is assumed to be $30 \mu \mathrm{m}$, consistent with the size separating cloud droplets and drizzle/rain drops in simulations of precipitation development through collision-coalescence (e.g., Berry and Reinhardt 1974). Only droplets smaller than the threshold are permitted to instantaneously evaporate because of saturation adjustment. In other words, $C$ in (1a) is based on the saturation adjustment that uses as input only the cloud droplet part of the spectrum, whereas $E$ in (1a) is derived from the shift of the drizzle/ rain part of the spectrum given the environmental subsaturation. It needs to be stressed that the above discussion applies only to the case of evaporation because the amount of cloud water allowed to evaporate in a single model time step in the saturation-adjustment procedure has to be limited by the available cloud water [see discussion following (7) in section 3a of GS90].

In the discrete system consisting of $\mathcal{N}$ bins (or classes) of drop sizes, the spectral density function for each bin $(i)$ [radius $r^{(i)}$ ] is defined as $f^{(i)}=n^{(i)} / \Delta r^{(i)}$, where $n^{(i)}$ is the concentration (per unit mass of dry air) of drops in the bin $i, \Delta r^{(i)}=r^{(i+1 / 2)}-r^{(i-1 / 2)}$ is the width of this bin, and the bin boundaries are defined as $r^{(i+1 / 2)}=\left[r^{(i+1)}+r^{(i)}\right] / 2$. This transforms the continuous Eq. (1c) into a system of $\mathcal{N}$ coupled equations (e.g., Morrison and Grabowski 2007):

$$
\begin{aligned}
& \frac{\partial f^{(i)}}{\partial t}+\frac{1}{\rho_{o}} \nabla \cdot\left\{\rho_{o}\left[\mathbf{u}-\mathbf{k} v_{t}^{(i)}\right] f^{(i)}\right\} \\
& =\left[\frac{\partial f^{(i)}}{\partial t}\right]_{\text {cond }}+\left[\frac{\partial f^{(i)}}{\partial t}\right]_{\text {act }}+\left[\frac{\partial f^{(i)}}{\partial t}\right]_{\text {coal }} \\
& \text { for } i=1, \ldots, \mathcal{N},
\end{aligned}
$$

where the first term on the rhs represents the condensation and evaporation terms $C$ and $E$ in (1c) (i.e., the transport of droplets/drops across the bin space due to their diffusional growth or evaporation) and, as in (1c), the second and third terms represent cloud droplet activation and growth by collision-coalescence, respectively. The total water mixing ratio in the discrete system is given by $Q=\sum_{i=1}^{\mathcal{N}} q_{i}^{(0)} f^{(i)} \Delta r^{(i)}$, where $q_{i}^{(0)}$ is the mass of a single droplet with radius $r^{(i)}$.

In a traditional bin scheme, the key is to first predict the supersaturation $S$ and subsequently to derive the shift of the spectral density function $f$ and the corresponding condensation rate $C$. In the hybrid bulk-bin scheme, this procedure is reversed: we first derive the condensation rate $C$ required to maintain water saturation and subsequently calculate the corresponding shift of the droplet spectrum. The shift of the spectrum is calculated using a bogus supersaturation $S^{*}$ that leads to the appropriate condensation rate $C$. The bogus supersaturation $S^{*}$ is derived from the expression of the condensation rate in the bin microphysics scheme, namely,

$$
C=4 \pi \rho_{w} A S^{*} \sum_{i=1}^{\mathcal{N}} f^{(i)} \Delta r^{(i)} r^{(i)} f_{\text {vent }}^{(i)} .
$$

Once the bogus supersaturation $S^{*}$ has been calculated given the bulk condensation rate $C$, the evolution of the droplet spectrum due to condensational growth is calculated as in the bin scheme, together with the growth by collision-coalescence. This strategy requires an important modification near the cloud boundaries, an aspect discussed in detail in section 4.

An important element of the hybrid approach is the activation of cloud droplets. In the bin scheme, the activation progresses gradually when the supersaturation increases and more droplets become activated. This process typically requires high spatial and temporal resolution (see discussions in Clark 1974 and Morrison and Grabowski 2008) to accurately capture the maximum supersaturation that determines the total number of activated droplets. This is why an activation parameterization is often used in cloud models that need to predict the number of activated droplets (e.g., models applying the double-moment bulk microphysics). Such parameterizations typically relate the number of activated droplets to the characteristics of cloud condensation nuclei (CCN; e.g., the chemical composition, size distribution, etc.) and the updraft strength (e.g., Twomey 1959; Ghan et al. 1993; Cohard et al. 1998). Such an approach has to be used in the hybrid scheme as well, and activated cloud droplets are inserted at the small droplet end of the spectral representation. The activation in the hybrid scheme occurs in a single time step when the cloud water first appears (i.e., when the bulk condensation rate is nonzero but there are no cloud droplets). Activation of cloud droplets used here applies a simple method detailed in the appendix. Note that additional activation of cloud droplets might occur inside the cloud above the cloud base when the supersaturation exceeds the value encountered by the air parcel near the cloud base (e.g., because of increasing updraft speed, washout of cloud droplets by raindrops, or entrainment). We implicitly assume that such instances can be appropriately handled by the activation parameterization.

\section{Validation of the bulk-bin scheme using the adiabatic parcel model}

As a validation of the hybrid approach, the scheme was adopted to the rising adiabatic parcel framework used in Grabowski and Wang (2009, hereafter GW09). The adiabatic parcel model solves equations for the 
temperature, pressure, and water vapor mixing ratio, as well as the set of discretized equations for the spectral density function inside an adiabatic air parcel rising with a constant vertical velocity. Except for the pressure equation, adiabatic parcel equations are as (1) except that the lhs terms are replaced with the time or space derivative $(d / d t \equiv w d / d z)$ and droplet/drop sedimentation is not considered (see GW09 for a detailed discussion). The microphysical processes include initial activation of cloud droplets and their growth by diffusion of water vapor and by collision-coalescence. We select maritime conditions because in such a case supersaturations are higher than in the continental case, and larger differences between the bin and hybrid approaches are expected. For the same reason a relatively strong updraft of $5 \mathrm{~m} \mathrm{~s}^{-1}$ is assumed.

The bin scheme used in GW09 provides a benchmark to which the hybrid scheme is compared. Both schemes apply exactly the same bin setup with a moderate number of bins (80; see the appendix for details). GW09's bin scheme includes neither Kelvin nor Rault effects and, as explained in the appendix, represents droplet activation in a relatively simple way. Since the hybrid scheme assumes the same number of activated droplets as the bin scheme, including Kelvin and Rault effects is not essential for the comparison. The main goal is to show the integrity of the computational approach used in the hybrid scheme, where changes of the droplet spectrum are driven by the bulk (i.e., saturation-adjustment) condensation rate. A 1-s time step is used in both the bin and hybrid schemes. Initial conditions are as in GW09, with $T(0)=288.16 \mathrm{~K}, p(0)=900 \mathrm{hPa}, q_{v}(0)=q_{v \mathrm{~s}}[T(0), p(0)]$ [i.e., $S(0)=0$ ], and $f^{(i)}(0)=0$ for $i=1, \ldots, \mathcal{N}$. Simulations proceed until the radar reflectivity factor (the sixth moment of the droplet size distribution) reaches $25 \mathrm{dBZ}$.

As discussed in GW09, upward motion of the air parcel causes initial condensation of cloud water. Once the radius of cloud droplets reaches about 10 microns, collisioncoalescence sets in and drizzle/rain starts to develop. The classical phases of the droplet spectral evolution due to collision-coalescence can be identified (e.g., Berry and Reinhardt 1974). First, collisions between cloud droplets of similar sizes widen the spectrum during the autoconversion phase. Later, during the accretion phase, already-present drizzle drops efficiently collect cloud droplets and the first raindrops appear. Overall, it takes about $9 \mathrm{~min}$ and about $2.7 \mathrm{~km}$ of the vertical displacement to develop radar reflectivity of $25 \mathrm{dBZ}$.

Results from bin and hybrid schemes are broadly consistent with results discussed in GW09 and the differences between the two schemes are small. For instance, the times that radar reflectivity reaches thresholds of $-20,-10,0,10$, and $20 \mathrm{dBZ}$ in the bin scheme are 0.93 ,
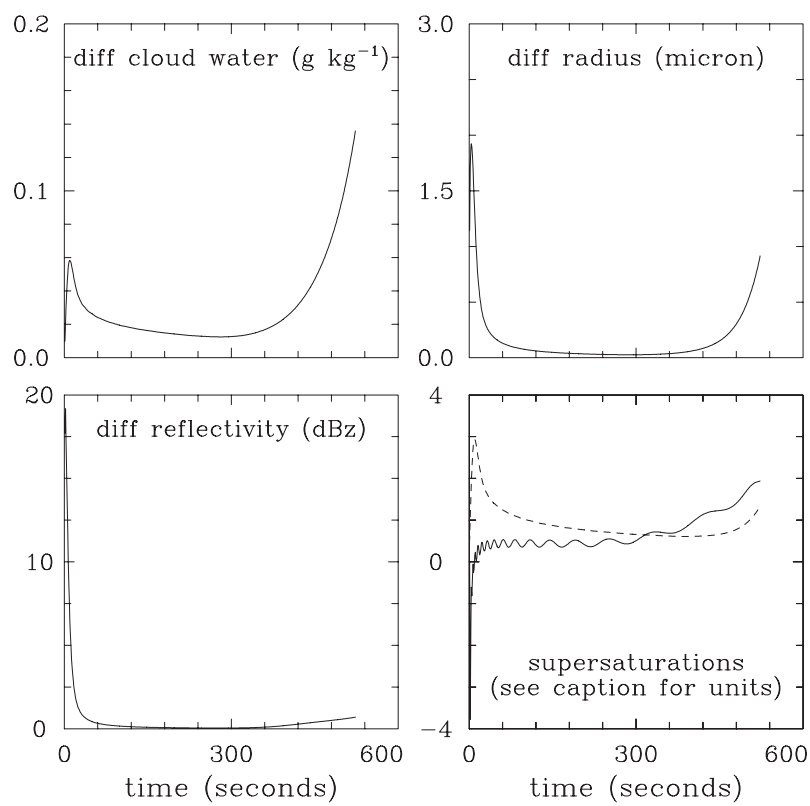

FIG. 1. Evolution of the differences between results from the bin and hybrid bulk-bin schemes for the adiabatic parcel simulations as in GW09. The upper panels and the lower left panel show the differences for the cloud water, mean volume radius, and radar reflectivity. The lower right panel shows the evolution of the supersaturation in bin (dashed) and hybrid (solid) schemes; the unit on the vertical axis is $0.5 \%$ and $0.01 \%$ for the bin and hybrid schemes, respectively.

$2.93,6.58,7.65$, and $8.42 \mathrm{~min}$, respectively. For the hybrid scheme, these are $0.90,2.92,6.57,7.62$, and $8.37 \mathrm{~min}$. To document the differences, Fig. 1 shows the evolution of the differences in cloud water, mean volume radius, and radar reflectivity between the bin and the hybrid bulkbin schemes, as well as the evolution of the supersaturation in both schemes. Note that the supersaturation in the hybrid scheme is derived from the temperature and water vapor mixing ratio and does not have to be exactly zero because of truncation errors in the bogus supersaturation approach used to calculate spectral transformations of cloud droplets. The differences in results from the two schemes are relatively small (for instance, a few hundredths of $\mathrm{g} \mathrm{kg}^{-1}$ for the cloud water) and are the largest early in the simulations because of different treatments of the activation. The difference in the cloud water toward the end of the simulations may seem significant, but this is still just a few percent of the total condensed water (which is about $5 \mathrm{~g} \mathrm{~kg}^{-1}$ at the end of the simulation). The evolution of the supersaturation in the hybrid scheme shows that the bogus supersaturation procedure to calculate shift of the spectrum leads to a small deviation of the bulk scheme from exact water saturation, typically a fraction of $0.01 \%$. This is much smaller than the supersaturation in the bin scheme, which 
reaches a maximum around $1.5 \%$ during activation and stays around $0.5 \%$ for the most of the simulation.

In summary, rising adiabatic parcel simulations show that representation of spectral changes of cloud droplets and drizzle/rain drops using the condensation rate predicted by the saturation adjustment is a valuable approach and, after being combined with the representation of advection in the physical space, can be used in multidimensional models of warm clouds.

\section{1D advection-condensation problem}

This section discusses application of the hybrid bulkbin scheme to the advection-condensation problem of GS90. The test highlights numerical problems that finitedifference schemes experience near cloud boundaries and demonstrates the need for a special approach when the hybrid bulk-bin microphysics scheme is combined with the finite-difference representation of advection in the physical space. In the context of the bin scheme, these numerical problems were noted in Grabowski (1989) and discussed in Stevens et al. (1996). Mitigation of these problems in models predicting supersaturation requires a special approach (Grabowski and Morrison 2008).

The 1D advection-condensation problem considers constant-velocity advection of a square-wave perturbation in the temperature, water vapor, and cloud water along a 1D grid. Physically, this represents either vertical (as in GS90) or horizontal (as in Stevens et al. 1996) advection of a cloud that is uniform in the direction perpendicular to the advective velocity (e.g., a vertical advection of a horizontally uniform cloud). In the case of horizontal advection, the analytic solution represents a simple translation of the initial square-wave cloud without any condensation or evaporation. The finite-difference approximation, on the other hand, results in some condensation and evaporation near cloud edges when the cloud boundary is advected over the grid. The net effect is the evaporation of some cloud near its original edge, which leads to the reduction of the width of initial perturbation and characteristic undershoots of the temperature because of evaporative cooling (e.g., Figs. $3 \mathrm{a}$ and $4 \mathrm{a}$ in GS90; Figs. 5 and 6 in Grabowski and Morrison 2008). For the vertical advection, there is condensation inside the cloud and the bulk condensation rate predicted by the saturation adjustment scheme shows overshoots near cloud edges.

We combined the hybrid microphysics scheme of condensation (i.e., no collision-coalescence and no droplet sedimentation) with a 1D finite-difference representation of the advection and bulk condensation. Such a system forms a convenient test bed before the hybrid bulk-bin microphysics scheme is included into a multidimensional cloud model. As in GS90, a 1D version of the multidi- mensional positive definite advection transport algorithm (MPDATA) scheme is used to represent advection of model variables in the physical space. A single time step in the $1 \mathrm{D}$ advection-condensation test (and in the multidimensional framework presented in the next section) using the hybrid scheme proceeds in the following way. First, the bulk variables $\left(\theta, q_{v}\right.$, and $q_{c}$; the latter is calculated from the spectral representation of the condensed water) are advected along the $1 \mathrm{D}$ grid and subsequently adjusted to maintain water saturation. This is followed by the advection along the $1 \mathrm{D}$ grid of all bins of the spectral representation of the cloud water. The difference between the local cloud water derived from bulk advectioncondensation $\left(q_{c}^{\text {bulk }}\right)$ and the cloud water obtained from the advected bins $\left(q_{c}^{\text {bin }}\right)$ is equal (after dividing by the model time step) to the condensation rate needed to maintain saturation. This condensation rate is used to calculate the bogus supersaturation $S^{*}$ in (3), which is subsequently used to derive the spectral change. If needed, instantaneous activation or deactivation of cloud droplets takes place, that is, when $q_{c}^{\text {bulk }}>0$ but $q_{c}^{\text {bin }}=0$ or when $q_{c}^{\text {bulk }}=0$ but $q_{c}^{\text {bin }}>0$, respectively. Derivation of the new bulk cloud water $q_{c}$ from the updated drop spectrum and adjustment of the water vapor mixing ratio to conserve total water completes the model time step.

The numerical setup of the advection-condensation test (the grid length, time step, etc.) is exactly as in GS90, as are the initial conditions (i.e., background profiles and square-wave perturbations). The initial cloud water perturbation is introduced to the hybrid scheme through a simple procedure in which the bin microphysics routine is called 10 times, each time increasing the cloud water content by $10 \%$ of its final value (i.e., the value that forms the initial condition for the test). This results in droplet activation in the first call and a gradual shift of the spectrum toward larger sizes in subsequent calls. The initial perturbations are then advected using the MPDATA routine as in GS90 with a vertical velocity of $2 \mathrm{~m} \mathrm{~s}^{-1}$. Since collision-coalescence processes are excluded from the advection-condensation test, $\mathcal{N}=30$ is assumed; this extends the spectral density function up to $48.7 \mu \mathrm{m}$ only.

Figure 2 shows the quasi-analytic solution using the hybrid model obtained with the time step corresponding to the Courant number of 1 (i.e., the advection scheme simply shifts the initial perturbation one grid increment in each time step). ${ }^{2}$ The upper two panels show the potential temperature and cloud water mixing ratio (which are exactly as the analytic solution shown in GS90), whereas

\footnotetext{
${ }^{2}$ We use the term "quasi-analytic" because the bulk condensation rate from the saturation adjustment (the same as in the analytic solution to the advection-condensation problem) is used in the numerical calculation of the spectral change.
} 

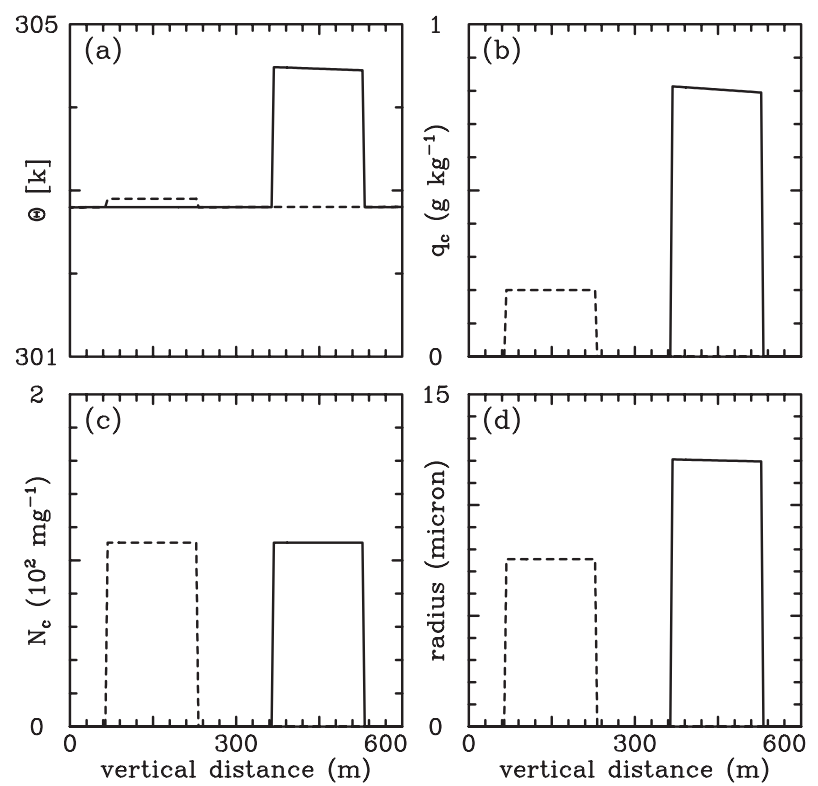

FIG. 2. Quasi-analytic solution of the GS90 advectioncondensation test (time step of $2 \mathrm{~s}$; Courant number equal to 1) using the hybrid bulk-bin scheme. The panels show (a) the potential temperature, (b) cloud water mixing ratio, (c) cloud droplet concentration, and (d) mean volume radius.

the bottom panels show the corresponding concentration and mean radius of cloud droplets. As anticipated, vertical advection of the initial perturbation results in an increase of the droplet size with unchanged droplet concentration. Droplet spectra are the same across the squarewave perturbation (not shown).

When the same problem is solved with a smaller time step (i.e., Courant number of 0.33 as in GS90), the solution suffers from significant problems as illustrated in Fig. 3. First, there are undershoots and overshoots of the temperature field near cloud edges and a small cloud water overshoot near the leading edge. These are exactly as in Fig. 4 of GS90. More importantly, the cloud droplets near the edges are significantly larger than inside the cloud (note a different vertical scale in the lower right panel of Fig. 3). These "radius overshoots" result from the dilution of the droplet concentration that leads to the "superadiabatic" growth; that is, the mean droplet radius becomes significantly larger than in the central adiabatic part of the perturbation. Inspection of the droplet spectra (not shown) documents that the spectra near cloud edges are wide and shifted toward larger sizes compared to the spectra away from the interface. This is a major problem that needs to be corrected.

The key feature of the numerical algorithm is the assumption that grid boxes near the interface with reduced droplet concentration are homogeneous, as illustrated in the upper part of Fig. 4. However, another interpre-
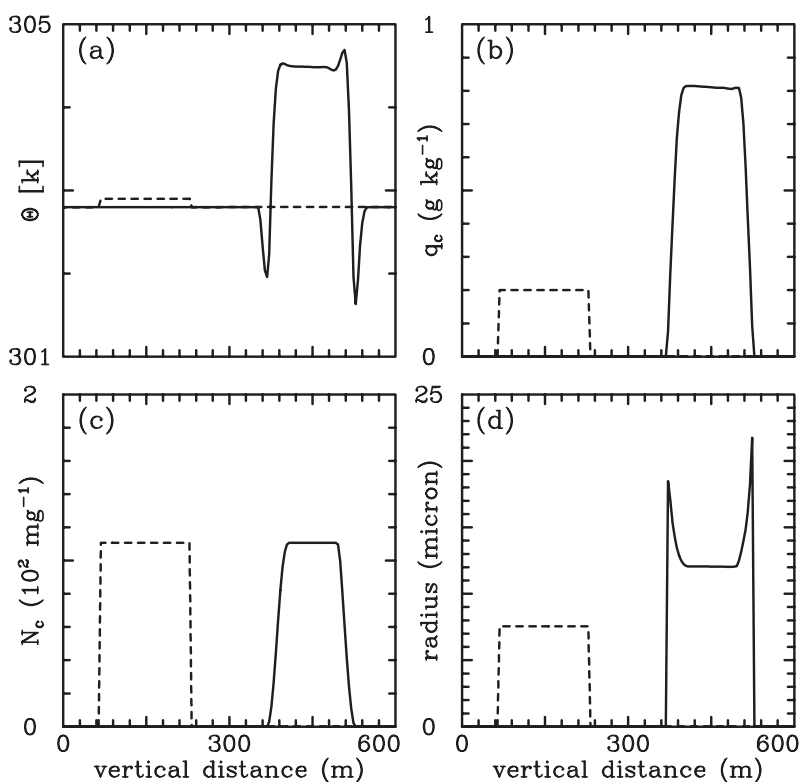

FIG. 3. As in Fig. 2, but using time step of $0.667 \mathrm{~s}$ (Courant number of 0.33 ). Note a different vertical scale on the lower right panel showing the mean droplet radius.

tation of the mean dilution near the interface might be that such grid boxes are partly cloudy, as illustrated by the lower part of Fig. 4. We will refer to such grid boxes as heterogeneous. When homogeneous and heterogeneous grid boxes are allowed in the numerical model, one then needs to include different approaches to represent spectral changes due to advection and condensation/ evaporation depending on whether the grid box is homogeneous and cloudy or heterogeneous and partly cloudy. This is illustrated in Fig. 5. If the grid box can be assumed to be homogeneous, then for the case of condensation/evaporation all droplets are exposed to the same sub- or supersaturation. In such a case, the condensation/evaporation shifts the spectral density function toward larger/smaller sizes as illustrated via changes from the initial spectrum (thick solid line) to the final spectrum (dashed line) in Fig. 5. However, if the grid box is heterogeneous (i.e., part of the grid box is cloudy and the rest is cloud free), then the advection and/or condensation/evaporation may lead to changes of the grid-averaged droplet concentration without affecting their size. For instance, a simple advection of a cloud into a partly cloudy grid box without any phase changes represents exactly the heterogeneous process: the grid box-averaged concentration of droplets increases without changing their mean size. For the condensation/ evaporation, the heterogeneous phase change implies a change of the fraction of the grid box occupied by the cloud with no change of cloud properties. Heterogeneous transformations are illustrated by spectral changes from 


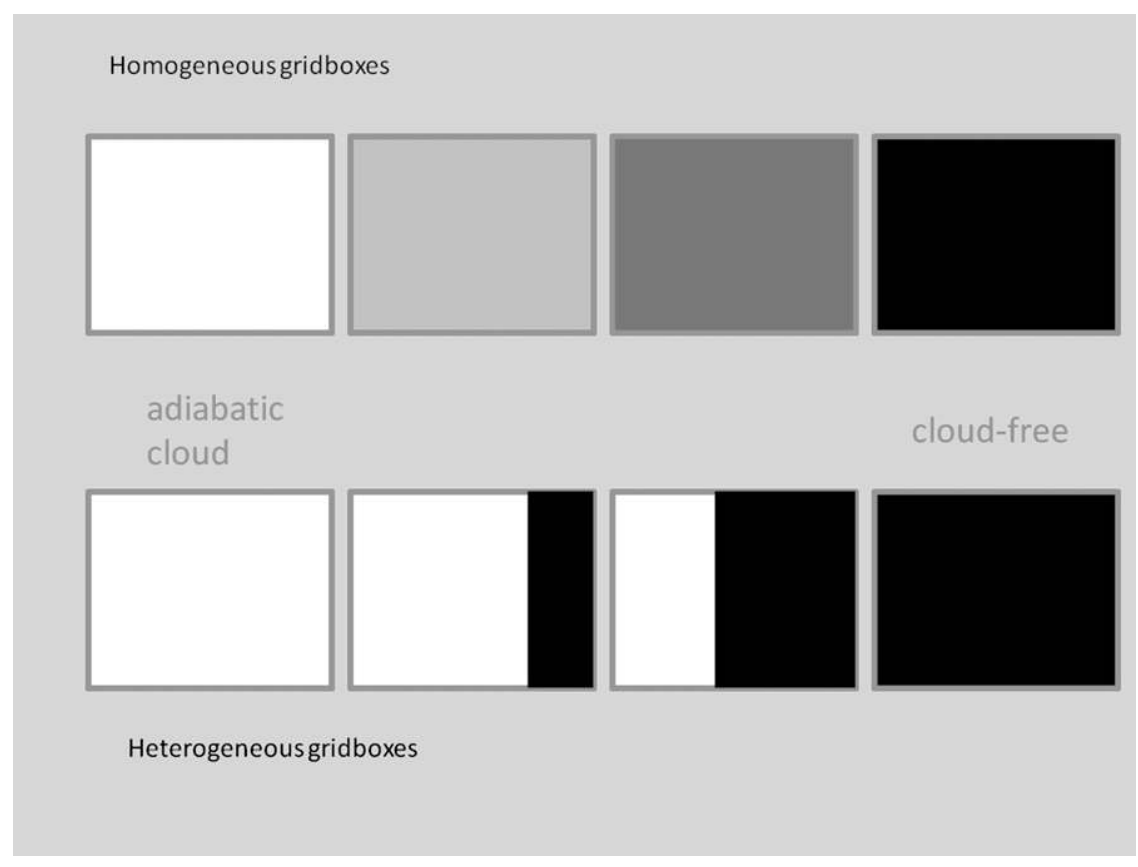

FIG. 4. Distribution of the cloud droplets in grid boxes near the cloud edge. The upper part shows the homogeneous case: the numerical scheme assumes that diluted grid boxes are uniformly filled with cloud droplets. The lower part shows the heterogeneous case: diluted grid boxes are assumed to be partially cloudy, with the cloudy part featuring the same local droplet concentration as the adiabatic fully cloudy grid boxes away from the cloud edge.

thick to thin solid lines in Fig. 5. They are simple to represent numerically because the spectral density function has to be multiplied by the ratio between the initial and the final cloud water mixing ratio $q_{c}^{\text {bulk }} / q_{c}^{\text {bin }}$. We emphasize that homogeneous and heterogeneous processes are introduced to the finite-difference representation of the advection-condensation problem to ensure physical consistency of the resulting algorithm. In particular, the heterogeneous evaporation described above is exactly what is referred to as the extremely inhomogeneous mixing of Baker and Latham (1979) and Baker et al. (1980). The extremely inhomogeneous mixing has a sound theoretical and observational foundation (e.g., Baker et al. 1980; Burnet and Brenguier 2007), which has nothing to do with numerical aspects discussed here.

The selection of the homogeneous versus heterogeneous spectral changes in the advection-condensation problem requires identification of heterogeneous grid boxes. This is accomplished by including an additional model variable, the fraction of the grid box volume with cloudy air $\beta$, as in Jarecka et al. (2009). The governing equation for $\beta$ is

$$
\frac{\partial \beta}{\partial t}+\frac{1}{\rho_{o}} \nabla \cdot\left(\rho_{o} \mathbf{u} \beta\right)=S_{\beta},
$$

where $S_{\beta}$ is the source/sink term. Since $\beta=0$ outside a cloud and $\beta=1$ inside, the source/sink term resets $\beta$ to unity (or to zero) when saturation adjustment produces cloud water in initially cloud-free grid box (or when saturation adjustment forces all cloud water to evaporate). Also, $S_{\beta}$ should modify $\beta$ for the heterogeneous grid box, with the updated $\beta$ related to the advected $\beta^{*}$ as

$$
\beta=C_{\beta} \beta^{*}, \quad C_{\beta} \equiv \frac{q_{c}^{\text {bulk }}}{q_{c}^{\text {bin }}},
$$

where the coefficient $C_{\beta}$ is the same as used in the heterogeneous transformation of the cloud droplet spectra. Note that $C_{\beta}$ has to be smaller or equal to $1 / \beta^{*}$ to ensure that $\beta \leq 1$. When $C_{\beta}=1 / \beta^{*}$, a heterogeneous grid box becomes homogeneous and there is still some cloud water left after heterogeneous change of the spectrum because $q_{c}^{\text {bulk }} / q_{c}^{\text {bin }}>1 / \beta^{*}$. In such a case, additional homogeneous transformation of the spectrum has to take place to ensure that the final cloud water in the bin model matches $q_{c}^{\text {bulk }}$.

Figures 6 and 7 show the results of GS90's test using the hybrid bulk-bin scheme and the homogeneous/ heterogeneous spectral changes based on the predicted $\beta$. The bulk solutions are obviously very close to those shown in Fig. 3, but the microphysical properties are now consistent with the quasi-analytic solution. In particular, neither the total droplet concentration nor the mean volume radius shows any significant artifacts, although 

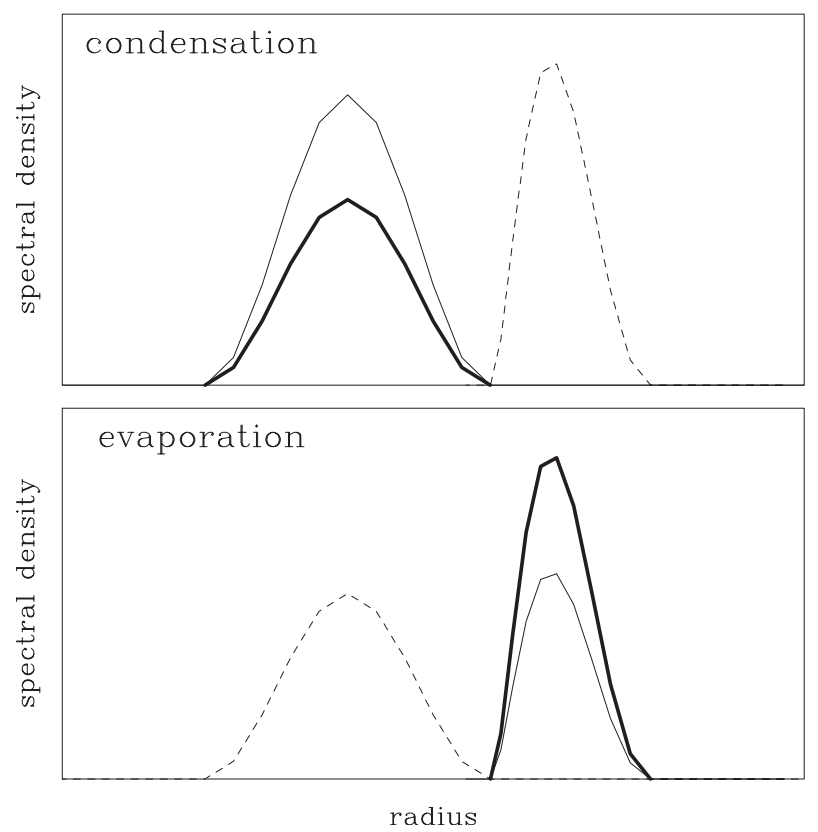

FIG. 5. Schematic illustration of the spectral change due to (top) condensation and (bottom) evaporation associated with homogeneous and heterogeneous processes. In each panel, the thick solid line shows the initial droplet spectral density function. The homogeneous condensation/evaporation results in the spectral density shown using the dashed line. The heterogeneous process results in the spectral density shown by the thin solid line.

the droplet radius is slightly reduced near cloud edges. It is our experience that the solution near the edges depends to some extent on the particular threshold of $\beta$ applied to distinguish between the homogeneous and heterogeneous grid boxes. In all examples shown in this paper, the grid box is assumed homogeneous when $\beta \geq$ 0.999. Decreasing this threshold to 0.95 improves the mean droplet size near the interface in the case shown in Fig. 6, but it degrades the solution (by adding small superadiabatic growth) in cases where the flow Courant numbers are smaller.

Figure 7 shows drop mass density distributions (spectra) of cloud droplets $d m(r) / d \log (r)[m(r)$ is the mass of droplets with radius $r$ per unit mass of dry air] near cloud edges. The upper (lower) row shows the spectra in grid locations near the upper (lower) interface. For the upper row, the next grid box to the right (i.e., for $k=132$ ) has no cloud water. For the lower row, the next grid box to the left (i.e., for $k=94$ ) has no cloud water either. Droplet spectra from the quasi-analytic solution are also shown for reference. The figure shows that the condensational growth using the homogeneous/heterogeneous approach results in droplet spectra near cloud edges that are consistent with the analytic solution, although some reduction of the mean droplet size, already shown in Fig. 6d, is apparent. The
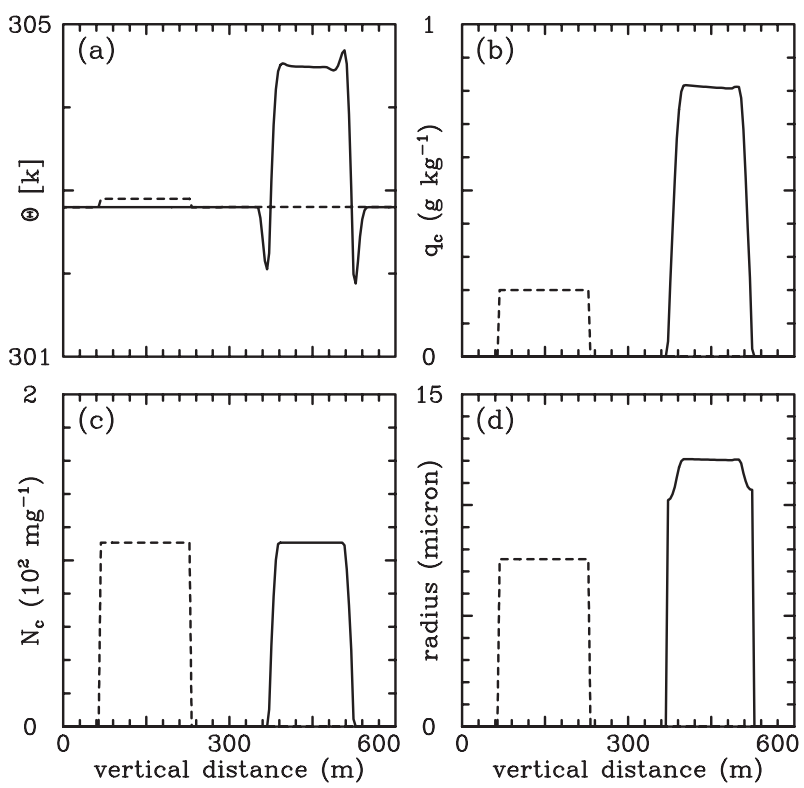

FIG. 6. As in Fig. 2, but using a time step of $0.667 \mathrm{~s}$ (Courant number of 0.33 ) and applying the homogeneous/heterogeneous condensation as discussed in text.

main inconsistency is the reduced droplet concentration in regions where numerical diffusion causes a gradual transition from the cloud to the cloud-free environment. In particular, no superadiabatic growth is simulated.

In summary, we propose here a relatively straightforward technique to provide a physically consistent approach to merge the hybrid bulk-bin microphysics scheme with the finite-difference representation of $1 \mathrm{D}$ advection in the physical space. The key aspect is to recognize the need for both homogeneous and heterogeneous changes of the cloud droplet spectra on, respectively, homogeneous and fully cloudy or heterogeneous and partly cloudy grid boxes. The additional prognostic model variable, the fraction of the grid box covered by cloudy air, $\beta$ (cf. Jarecka et al. 2009), guides the selection of either the homogeneous or heterogeneous spectral change. Extension of this approach to the multidimensional framework and for precipitating clouds follows naturally. In the case of precipitating clouds, the homogeneous/heterogeneous spectral changes discussed in this section apply only to the cloud water part of the spectrum.

\section{Application of the bulk-bin scheme to the cloud model}

In this section, we present an application of the hybrid bulk-bin scheme to an idealized problem of 2D moist precipitating convection. In this problem, an initially circular moist warm bubble rises in the stratified environment and encounters a layer of stronger stability that arrests its vertical motion. The rise of the initial perturbation 

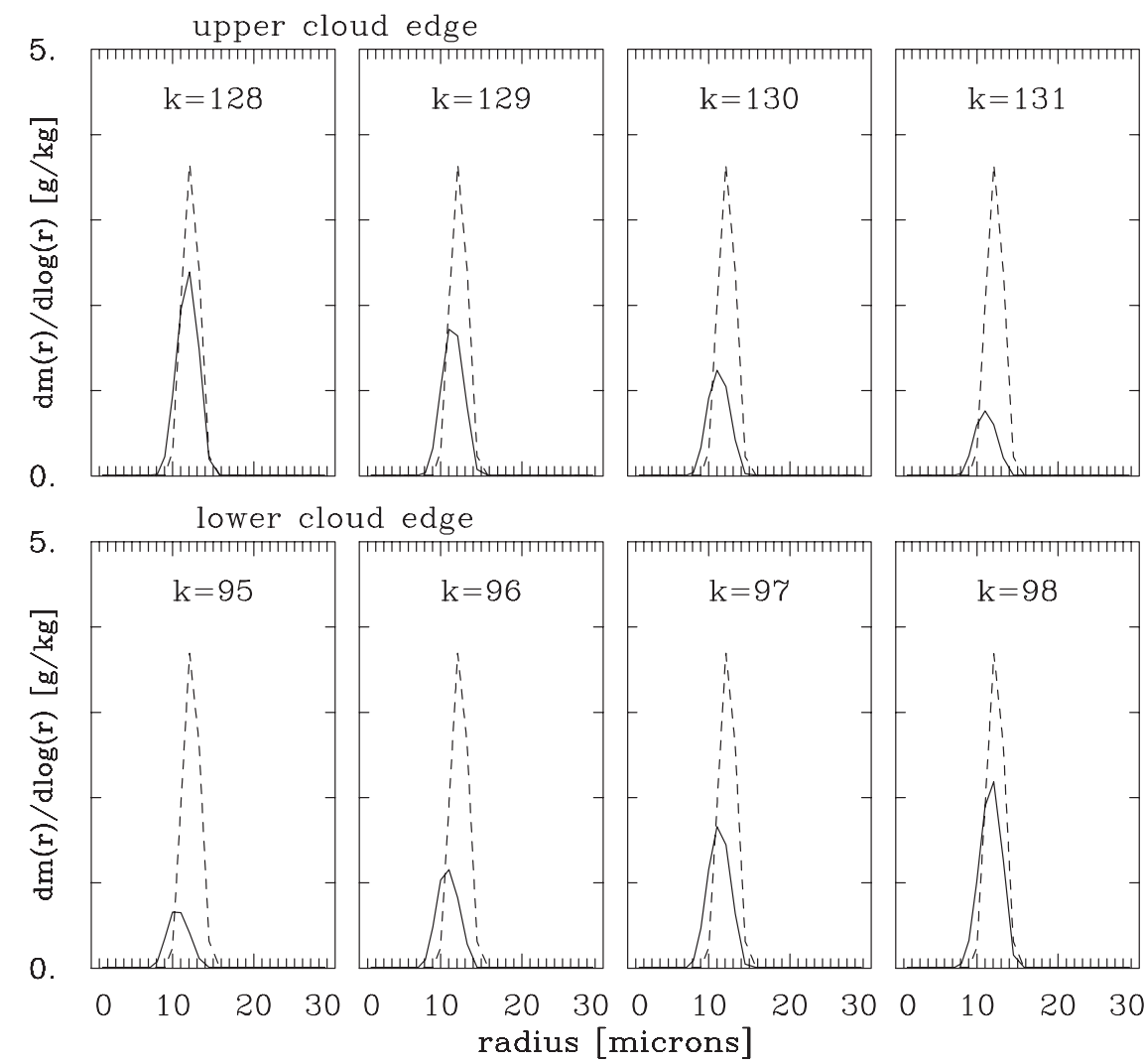

FIG. 7. Drop mass density distributions of cloud droplets near cloud edges from the simulation in Fig. 6 for (top) the upper and (bottom) lower cloud edge. Quasi-analytic distributions are shown as dashed lines.

results in the formation of a cloud and the development of drizzle/rain, with the latter falling out of the cloud and evaporating in subsaturated air beneath.

In contrast to simulations described in previous sections, sedimentation of both cloud droplets and raindrops are now included in the model physics. This requires a modification of the bulk scheme used in the hybrid model because a traditional bulk scheme typically excludes sedimentation of the bulk cloud water $q_{c}$. The approach used here is to allow $q_{c}$ at each spatial location to fall with mass-averaged terminal velocity of cloud water bins (i.e., bins up to $30 \mu \mathrm{m}$ ). Because of a relatively low spatial resolution of the $2 \mathrm{D}$ simulation presented here (grid length of $50 \mathrm{~m}$ ), this modification has a small impact on model results (i.e., the results differ little depending whether the sedimentation of cloud water is included or excluded). However, since the sedimentation of cloud water has been shown to have considerable impact on entrainment in stratocumulus-topped boundary layers (e.g., Ackerman et al. 2004; Bretherton et al. 2007), this process is included in the bulk-bin cloud model used here.

The dynamic model is a simple 2D flow model based on the anelastic semi-Lagrangian/Eulerian 3D model
EULAG documented in Smolarkiewicz and Margolin (1997). The 2D model was used as the superparameterization in simulations described in Grabowski and Smolarkiewicz (1999) and Grabowski (2001, 2004). It was also used as a stand-alone model in Grabowski (2006) and in Morrison and Grabowski (2008). The 2D computational domain, $5 \times 5 \mathrm{~km}^{2}$, is covered with a uniform grid of $50 \mathrm{~m}$ in both horizontal and vertical directions. Lateral boundaries are periodic and rigid boundaries are assumed at the surface and at $5 \mathrm{~km}$. Model time step is $2 \mathrm{~s}$.

The environmental stability is $(1 / \theta) d \theta / d z=1.3 \times$ $10^{-5} \mathrm{~m}^{-1}$ in the lower half of the domain and $3.0 \times$ $10^{-5} \mathrm{~m}^{-1}$ in the upper half. Relative humidity increases linearly from $75 \%$ at the surface to $95 \%$ at $2.5 \mathrm{~km}$ and is $95 \%$ above. The horizontal wind with a weak vertical shear is imposed, increasing linearly from 0 at the surface to $2 \mathrm{~m} \mathrm{~s}^{-1}$ at $5 \mathrm{~km}$. The air temperature and pressure at $z=0$ are $300 \mathrm{~K}$ and $1010 \mathrm{hPa}$. The initial circular perturbation (the bubble) is centered at $x=1.67 \mathrm{~km}$ and $z=1.5 \mathrm{~km}$. Inside the bubble, the temperature is increased by $0.5 \mathrm{~K}$ within the radius of $0.6 \mathrm{~km}$ and the relative humidity is set to $95 \%$. These perturbations 

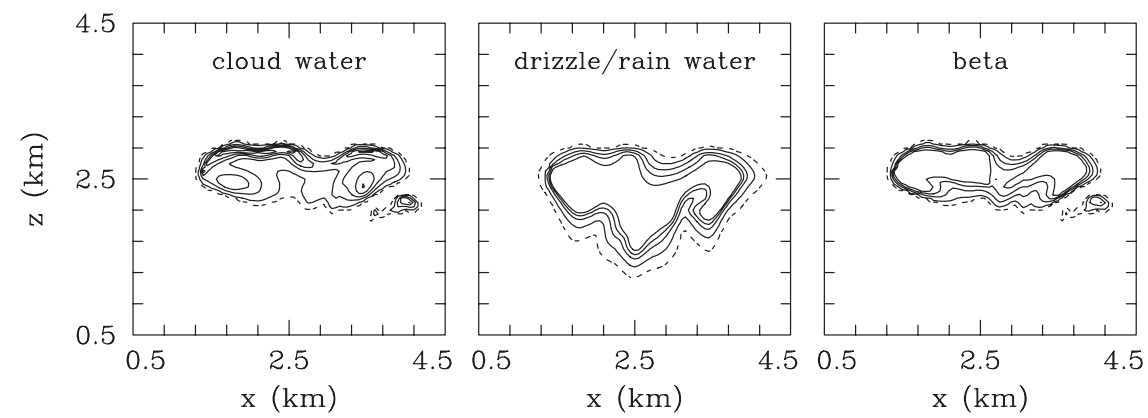

FIG. 8. Fields of the (left) cloud water and (middle) rainwater mixing ratios and (right) the fraction of the grid box occupied by the cloudy air $\beta$ for the rising thermal simulation at $t=$ $20 \mathrm{~min}$. Dashed contours are for values of $0.01 \mathrm{~g} \mathrm{~kg}^{-1}$ for the mixing ratios and 0.01 for $\beta$. Solid contours are drawn with contour interval of $0.1 \mathrm{~g} \mathrm{~kg}^{-1}$ for the mixing ratios and 0.1 for $\beta$; the first contour is for the value corresponding to the contour interval.

decrease linearly toward the environmental values over a 100-m-deep transition layer. No velocity perturbations are initially imposed.

The initial perturbations of the temperature and moisture force the rising motion within the bubble. Cloud water first appears between 2 and 3 min of the model simulation, with the local concentration of activated cloud droplets dependent on the model-predicted local vertical velocity as explained in the appendix. The maximum vertical velocity, close to $4 \mathrm{~m} \mathrm{~s}^{-1}$, is reached around 11th minute, with only a trace (below $0.01 \mathrm{~g} \mathrm{~kg}^{-1}$ ) of drizzle/ rain present at this time. After several more minutes, however, the drizzle/rain mixing ratio reaches about $2 \mathrm{~g} \mathrm{~kg}^{-1}$. Drizzle/rain falls out of the cloud and reaches the ground around $t=24 \mathrm{~min}$.

Figures 8 and 9 show selected model fields at $t=$ $20 \mathrm{~min}$. Figure 8 shows cloud water and drizzle/rainwater mixing ratios, as well as the cloud fraction $\beta$ near the center of the computational domain. The mixing ratios come from the integration of appropriate parts of the drop size distributions. Since sedimentation velocity of cloud droplets is small, the cloud water stays close to the temperature perturbation and is largest near the cloud top. In contrast, drizzle/rain drops feature significant sedimentation velocity and the drizzle/rain field extends significantly below the cloud. As anticipated, cloud fraction is close to one inside the cloud. These results are similar to a traditional bulk microphysics model (not shown), except for the rain field, which develops earlier in the bulk model simulation (the rain mixing ratio reaches $0.2 \mathrm{~g} \mathrm{~kg}^{-1}$ around $t=5$ min for the bulk model and only around $t=13 \mathrm{~min}$ in the bulk-bin model). ${ }^{3}$

\footnotetext{
${ }^{3}$ The bulk model used here applies Grabowski (1998) warm-rain microphysics assuming droplet concentration and relative dispersion [both needed for the autoconversion parameterization; cf. Eq. (8a) therein] of $100 \mathrm{~cm}^{-1}$ and 0.33 , respectively.
}

Figure 9 shows examples of the drop mass spectra in the center of the domain $(x=2.5 \mathrm{~km})$ and at heights of $z=2.0$ and $2.5 \mathrm{~km}$ at the same time as Fig. 8 (20 min). The selected locations correspond to either a grid point inside the cloud with some drizzle/rain or to a point inside a precipitation shaft beneath the cloud. Within the cloud, both cloud droplets and drizzle drops coexist and a characteristic minimum around 40-micron radius separates mass distributions of cloud droplets and drizzle drops. The maximum of the drizzle/rain part of the
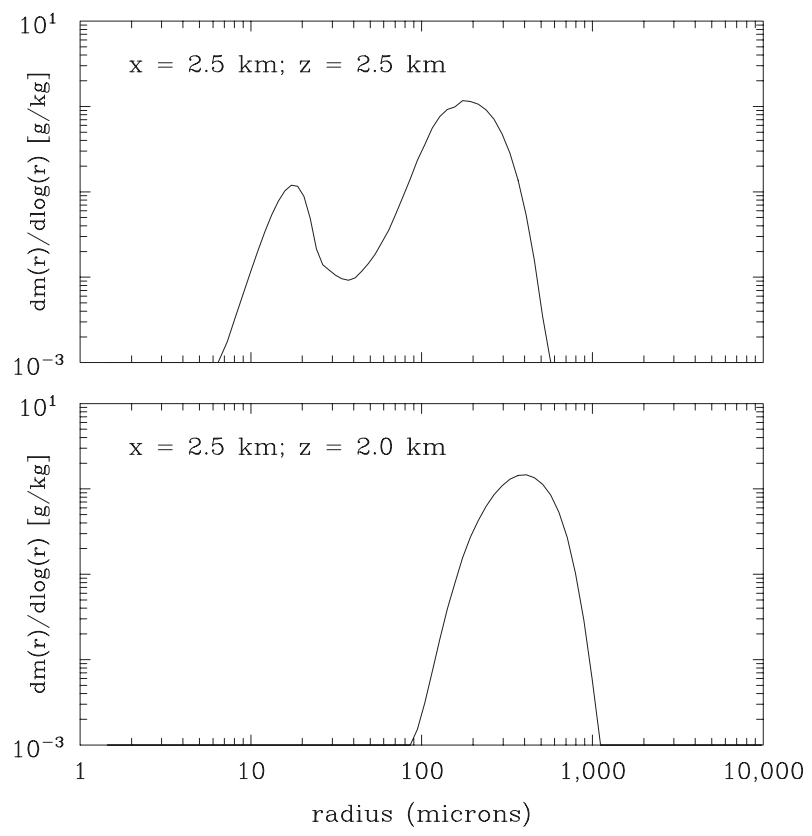

FIG. 9. Examples of the drop mass density distributions for two selected locations at $x=2.5 \mathrm{~km}$ and $z=$ (top) 2.5 and (bottom) $=$ 2.0; and for the snapshots of model solutions shown in Fig. 8. The points are in the center of the domain $(x=2.5 \mathrm{~km})$, either inside the cloud $(z=2.5 \mathrm{~km})$ or inside the rain shaft below the cloud $(z=2.0 \mathrm{~km})$. 
distribution is around 200 microns. Only drizzle/rain drops are present below the cloud. The maximum of the spectrum is around 400 microns and the distribution extends to sizes above $1 \mathrm{~mm}$, arguably because of the effects of drop self-collection within the rain shaft (Berry and Reinhardt 1974).

The above results document consistency of the hybrid bulk-bin scheme and its applicability to practical problems of warm cloud dynamics and microphysics.

\section{Summary}

This paper presents a hybrid approach to simulate warm-rain cloud microphysics that merges methodologies used in bulk and bin schemes. The bulk scheme assumes that cloud is always at water saturation and the condensation rate is predicted by the saturation adjustment. In the bin scheme, on the other hand, the condensation rate is derived from the predicted supersaturation field and the corresponding change of the droplet spectrum. The main idea behind the hybrid approach is to use the bulk condensation rate predicted by the saturation adjustment to evolve the spectrum of cloud droplets. Such an approach was used previously in the $b^{2}$ scheme of Brenguier and Grabowski (1993), but it is extended here to include collisional growth of cloud droplets. When the cloud water is first formed, the activation scheme inserts droplets at the small-droplet end of the spectral representation. Subsequent condensation shifts the spectrum toward larger sizes. The bulk condensation rate affects the cloud droplet part of the spectrum, with drizzle/rain drops evaporating in subsaturated conditions outside a cloud, on a finite time scale as in a traditional bin scheme.

An important feature of the hybrid scheme is that the details of the droplet activation are not considered. The scheme assumes that activation takes place in a single time step when the cloud water first appears. In contrast, traditional bin schemes attempt to resolve droplet activation, when the supersaturation builds up near the cloud base and progressively smaller $\mathrm{CCN}$ are activated. However, resolving droplet activation requires high vertical resolution (e.g., Clark 1974; Morrison and Grabowski 2008), often significantly larger than what can be afforded in large-eddy simulations. The adiabatic rising parcel simulations discussed in section 3 show that the largest differences between the hybrid and bin schemes occur exactly during droplet activation in the bin scheme, as one might anticipate. It follows that the hybrid scheme is best suited for cloud models with relatively low vertical resolution (say, a grid length of several tens of meters or larger) when details of the droplet activation are only poorly resolved (or not resolved at all, as in simulations of deep convection when model vertical grid length is
$100 \mathrm{~m}$ or larger). In addition, activation of cloud droplets above the cloud base (e.g., because of increasing updraft speed, washout of cloud droplets, or entrainment) needs to be allowed as well. In general, representation of droplet activation is an important feature of the hybrid scheme, an aspect not addressed in this paper.

Problems with predicting cloud properties near cloud edges have been long recognized in cloud modeling (e.g., Klaassen and Clark 1985; Grabowski 1989; GS90; Stevens et al. 1996; Grabowski and Morrison 2008). In the case of the bulk scheme, these numerical problems are in the form of condensation rate over- and undershoots. Since the hybrid scheme applies the bulk condensation rate, a special approach is needed to provide physically consistent solutions. An approach presented in this paper considers grid boxes near cloud edges to be partly cloudy and introduces different spectral changes over homogeneous fully cloudy and heterogeneous partly cloudy grid boxes. For the homogeneous growth, the spectral changes are as in a traditional bin scheme. For the heterogeneous grid boxes, however, heterogeneous changes of the droplet spectra are introduced (cf. Fig. 5). The physical interpretation of such changes is that advection/condensation in the heterogeneous case results in the change of the fraction of the grid box occupied by the cloudy air $\beta$. A prognostic equation for $\beta$ guides the selection of either homogeneous or heterogeneous spectral changes.

The homogeneous/heterogeneous approach to spectral changes can also be used in conjunction with other warm-rain schemes, such as a traditional bin scheme or a double-moment bulk scheme. The key is to modify the scheme physics to distinguish between fully cloudy and partly cloudy grid boxes. In the case of the bin microphysics, prediction of the supersaturation field, the key component of the scheme, should proceed differently for fully cloudy and partly cloudy grid boxes. In the double-moment warm-rain scheme (e.g., Morrison and Grabowski 2007, 2008) homogeneous processes represent changes of the droplet radius without affecting droplet concentration, whereas the heterogeneous processes change the droplet concentration without affecting the size. Applying the homogeneous/heterogeneous approach guided by the predicted $\beta$ would arguably be sufficient to remove cloud-edge supersaturation overshoots in the bin scheme (e.g., Grabowski 1989) as well as in the double-moment scheme (Grabowski and Morrison 2008). Finally, application of the bulk-bin scheme to realistic simulation of clouds requires development of an approach to represent various microphysical mixing scenarios (i.e., from the homogeneous mixing to the extremely inhomogeneous mixing; e.g., Burnet and Brenguier 2007; Andrejczuk et al. 2009) for 
the parameterized subgrid-scale turbulent exchange. These aspects are pursued in ongoing research and will be reported in forthcoming publications.

Acknowledgments. This work was completed during WWG's collaborative visit to CNRM supported by the CNRM's Research Visitor Fellowship. WWG was also partially supported by the NOAA Grant NA08OAR4310543 and the DOE ARM Grant DEFG02-08ER64574. Comments on the manuscript by Hugh Morrison are acknowledged.

\section{APPENDIX}

\section{Numerical Details of the Bin and Hybrid Bulk-Bin Schemes}

Both the bin and hybrid schemes used in the paper apply a linear-mass doubling grid that combines the linear grid with a grid often used in collision-coalescence studies in which the drop mass doubles every $s$ bins. The radius $r_{i}$ (in $\mu \mathrm{m}$ ) is obtained as

$r_{i}=(i-1) \alpha+\left(\frac{3 m_{i}}{4 \pi \rho_{w}}\right)^{1 / 3}$ for $i=1, \ldots, \mathcal{N}$,

where the mass $m_{i}$ is given by the recurrence $m_{i} / m_{i-1}=$ $2^{1 / s}$ and $m_{0}$ is taken as the mass of a droplet with $1-\mu \mathrm{m}$ radius [the second term on rhs of (4) needs to be converted into microns before it is added to the first term]. We use $\mathcal{N}=80, \alpha=0.5 \mu \mathrm{m}$, and $s=2$ in the rising parcel simulation (section 3) and 2D cloud model simulation (section 5). For the advection-condensation problem in section 4, the same grid is used except that it covers only cloud droplet part of the spectrum using $\mathcal{N}=30$.

The activation of cloud droplets in the bin scheme is treated in a manner similar to other bin schemes (e.g., Clark 1974; Hall 1980; Grabowski 1989; Stevens et al. 1996) and it is assumed that activated droplets are added to the first size bin. The number of activated $\mathrm{CCN}, N_{\mathrm{CCN}}$, is related to the supersaturation $S$ through a traditional expression (e.g., Twomey 1959; see also Pruppacher and Klett 1997):

$$
N_{\mathrm{CCN}}=C_{0}(100 S)^{k} \text {, }
$$

where $C_{0}$ and $k$ are coefficients determining the characteristics of the CCN. Herein, we use the clean maritime conditions with $C_{0}=120 \mathrm{mg}^{-1}$ and $k=0.4$. Equation (A2) is used in the bin scheme in the following way. At every time step, the value of the predicted supersaturation $S$ is compared to the maximum supersat- uration $S_{\max }$ experienced by the parcel in the past. If $S>$ $S_{\max }$, then additional condensation nuclei have to be activated and their number is derived as $\Delta n=C_{0}(100 S)^{k}-$ $C_{0}\left(100 S_{\max }\right)^{k}$. Subsequently, the spectral density function in the first bin is increased by $\Delta n / \Delta r^{(1)}$ and $S_{\max }$ takes the value of $S$. Such a simple approach results in realistic predictions of the droplet concentration, but not necessary spectral characteristics of the droplet spectrum just after activation, as illustrated in GW09.

Activation needs to be parameterized in the hybrid scheme. The "parameterization" used in all simulations described in this paper simply assumes that the concentration of activated droplets is equal to the concentration predicted by the adiabatic parcel bin model given the vertical velocity and selected aerosol characteristics [i.e., parameters $C_{0}$ and $k$ in (A2)]. This gives the concentration of about $51,93,111,140$, and $167 \mathrm{mg}^{-1}$ for vertical velocity of $0.1,1.0,2.0,5.0$, and $10.0 \mathrm{~m} \mathrm{~s}^{-1}$, respectively. Once the number of activated droplets is established, they are inserted into the bin representation in the following way. First, the lowest bin is identified that gives too much cloud water if all activated droplets were inserted there. Subsequently, the droplets are uniquely partitioned between that bin and its smaller neighbor so that the resulting cloud water matches the required amount.

In previous studies (e.g., Morrison and Grabowski 2007; GW09), condensation/evaporation of cloud droplets as well as evaporation of drizzle/rain drops were calculated using the 1D MPDATA advection scheme of Smolarkiewicz (1984). This typically required substepping (i.e., applying the scheme in several iterations with a smaller time step) because of the stability criteria for the Eulerian advection. Here, we apply an approach combining the analytic Lagrangian solution of the condensational growth with remapping of the spectral distribution onto the original radius grid using piecewise linear functions. Overall, this technique is similar to that used by Simmel and Wurzler (2006; section 3.1.2 and references therein). The analytic Lagrangian solution to the condensational growth considers evolution of the bin boundaries according to the droplet growth equation (i.e., $r^{2}=r_{o}^{2}+2 A S \Delta t$, where $r_{o}$ and $r$ are the initial and final radii given the supersaturation $S$ and the time increment $\Delta t$ ) and uses the number of droplets within each modified bin to derive the updated spectral density on the modified radius grid. To remap the updated spectral density back to the original grid, the spectral density function within each shifted bin is assumed to be a linear function of the radius. Unless no droplets are present in the bin, the slope of the linear function is derived from the values of the spectral distribution in neighboring bins using centered differences, and the intercept 
parameter is derived using the slope and the known number of droplets in the bin. In the final step, the piecewise linear functions are integrated along the original bin grid to obtain the number of droplets in each original bin, from which the updated spectral density function is derived. Such an approach conserves by design the total number of droplets and does not have any time step restrictions. When compared to 1D MPDATA, the new scheme provides similar results when short time steps dictated by the stability of the Eulerian advection are used. With longer time steps, the solutions are improved even further.

The numerical treatment of coalescence is the same as in Morrison and Grabowski (2007) and GW09. It involves the linear flux method of Bott (1998) and excludes droplet breakup.

\section{REFERENCES}

Ackerman, A. S., M. P. Kirkpatrick, D. E. Stevens, and O. B. Toon, 2004: The impact of humidity above stratiform clouds on indirect climate forcing. Nature, 432, 1014-1017.

Andrejczuk, M., W. W. Grabowski, S. P. Malinowski, and P. K. Smolarkiewicz, 2009: Numerical simulation of cloud-clear air interfacial mixing: Homogeneous versus inhomogeneous mixing. J. Atmos. Sci., 66, 2493-2500.

Baker, M. B., and J. Latham, 1979: The evolution of droplet spectra and the rate of production of embryonic raindrops in small cumulus clouds. J. Atmos. Sci., 36, 1612-1615.

— , R. G. Corbin, and J. Latham, 1980: The influence of entrainment on the evolution of cloud droplet spectra: I. A model of inhomogeneous mixing. Quart. J. Roy. Meteor. Soc., 106, 581-598.

Berry, E. X., and R. L. Reinhardt, 1974: An analysis of cloud drop growth by collection: Part I. Double distributions. J. Atmos. Sci., 31, 1814-1824.

Bott, A., 1998: A flux method for the numerical solution of the stochastic collection equation. J. Atmos. Sci., 55, 2284-2293.

Brenguier, J.-L., and W. W. Grabowski, 1993: Cumulus entrainment and cloud droplet spectra: A numerical model within a twodimensional dynamical framework. J. Atmos. Sci., 50, 120-136.

Bretherton, C. S., P. N. Blossey, and J. Uchida, 2007: Cloud droplet sedimentation, entrainment efficiency, and subtropical stratocumulus albedo. Geophys. Res. Lett., 34, L03813, doi:10.1029/ 2006GL027648.

Burnet, F., and J. L. Brenguier, 2007: Observational study of the entrainment-mixing process in warm convective clouds. J. Atmos. Sci., 64, 1995-2011.

Clark, T. L., 1973: Numerical modeling of the dynamics and microphysics of warm cumulus convection. J. Atmos. Sci., 30, 857-878.

- 1974: On modelling nucleation and condensation theory in Eulerian spatial domain. J. Atmos. Sci., 31, 2099-2117.

Cohard, J.-M., J.-P. Pinty, and C. Bedos, 1998: Extending Twomey's analytical estimate of nucleated cloud droplet concentrations from CCN spectra. J. Atmos. Sci., 55, 3348-3357.

Feingold, G., S. Tzivion, and Z. Levin, 1988: Evolution of raindrop spectra. Part I: Solution to the stochastic collection/breakup equation using the method of moments. J. Atmos. Sci., 45, 3387-3399.
_ B. Stevens, W. R. Cotton, and R. L. Walko, 1994: An explicit cloud microphysics/LES model designed to simulate the Twomey effect. Atmos. Res., 33, 207-233.

Ghan, S. J., C. C. Chuang, and J. E. Penner, 1993: A parameterization of cloud droplet nucleation. Part I: Single aerosol type. Atmos. Res., 30, 197-221.

Grabowski, W. W., 1989: Numerical experiments on the dynamics of the cloud-environment interface: Small cumulus in a shearfree environment. J. Atmos. Sci., 46, 3513-3541.

_ 1998: Toward cloud resolving modeling of large-scale tropical circulations: A simple cloud microphysics parameterization. J. Atmos. Sci., 55, 3283-3298.

- 2001: Coupling cloud processes with the large-scale dynamics using the cloud-resolving convection parameterization (CRCP). J. Atmos. Sci., 58, 978-997.

_ 2004: An improved framework for superparameterization. J. Atmos. Sci., 61, 1940-1952.

, 2006: Indirect impact of atmospheric aerosols in idealized simulations of convective-radiative quasi equilibrium. J. Climate, 19, 4664-4682.

- 2007: Representation of turbulent mixing and buoyancy reversal in bulk cloud models. J. Atmos. Sci., 64, 3666-3680.

— approximations to the advection-condensation problem. Mon. Wea. Rev., 118, 2082-2098.

$\longrightarrow$, and —, 1999: CRCP: A cloud-resolving convection parameterization for modeling the tropical convecting atmosphere. Physica D, 133, 171-178.

_ , and H. Morrison, 2008: Toward the mitigation of spurious cloud-edge supersaturation in cloud models. Mon. Wea. Rev., 136, 1224-1234.

_ , and L.-P. Wang, 2009: Diffusional and accretional growth of water drops in a rising adiabatic parcel: Effects of the turbulent collision kernel. Atmos. Chem. Phys., 9, 2335-2353.

Hall, W. D., 1980: A detailed microphysical model within a twodimensional dynamic framework: Model description and preliminary results. J. Atmos. Sci., 37, 2486-2507.

Jarecka, D., W. W. Grabowski, and H. Pawlowska, 2009: Modeling of subgrid-scale mixing in large-eddy simulation of shallow convection. J. Atmos. Sci., 66, 2125-2133.

Kessler, E., 1969: On the Distribution and Continuity of Water Substance in Atmospheric Circulations. Meteor. Monogr., No. 32, Amer. Meteor. Soc., 84 pp.

Khairoutdinov, M., and Y. Kogan, 2000: A new cloud physics parameterization in a large-eddy simulation model of marine stratocumulus. Mon. Wea. Rev., 128, 229-243.

Klaassen, G. P., and T. L. Clark, 1985: Dynamics of the cloudenvironment interface and entrainment in small cumuli: Twodimensional simulations in the absence of ambient shear. J. Atmos. Sci., 42, 2621-2642.

Kogan, Y. L., 1991: The simulation of a convective cloud in a 3-D model with explicit microphysics. Part I: Model description and sensitivity experiments. J. Atmos. Sci., 48, 1160 1189.

Meyers, M. P., R. L. Walko, J. Y. Harrington, and W. R. Cotton, 1997: New RAMS cloud microphysics parameterization. Part II: The two-moment scheme. Atmos. Res., 45, 3-39.

Milbrandt, J. A., and M. K. Yau, 2005: A multimoment bulk microphysics parameterization. Part I: Analysis of the role of the spectral shape parameter. J. Atmos. Sci., 62, 3051-3064.

Morrison, H., and W. W. Grabowski, 2007: Comparison of bulk and bin warm-rain microphysics models using a kinematic framework. J. Atmos. Sci., 64, 2839-2861. 
- and - 2008: Modeling supersaturation and subgrid-scale mixing with two-moment bulk warm microphysics. J. Atmos. Sci., 65, 792-812.

— , J. A. Curry, and V. I. Khvorostyanov, 2005: A new doublemoment microphysics parameterization for application in cloud and climate models. Part I: Description. J. Atmos. Sci., 62, 1665-1677.

Pruppacher, H. R., and J. D. Klett, 1997: Microphysics of Clouds and Precipitation. Kluwer Academic, 954 pp.

Seifert, A., and K. D. Beheng, 2001: A double-moment parameterization for simulating autoconversion, accretion and selfcollection. Atmos. Res., 59-60, 265-281.

Simmel, M., and S. Wurzler, 2006: Condensation and activation in sectional cloud microphysical models. Atmos. Res., 80, 218-236.
Smolarkiewicz, P. K., 1984: A fully multidimensional positive definite advection transport algorithm with small implicit diffusion. J. Comput. Phys., 54, 325-362.

__, and L. G. Margolin, 1997: On forward-in-time differencing for fluids: An Eulerian/semi-Lagrangian nonhydrostatic model for stratified flows. Atmos.-Ocean, 35, 127-152.

Smoluchowski, M., 1916: Drei Vorträge über Diffusion, Brownsche Molekularbewegung und Koagulation von Kolloidteilchen. Phys. Z., 17, 557-585.

Stevens, B., R. L. Walko, W. R. Cotton, and G. Feingold, 1996: The spurious production of cloud-edge supersaturation by Eulerian models. Mon. Wea. Rev., 124, 1034-1041.

Twomey, S., 1959: The nuclei of natural cloud formation. Part II: The supersaturation in natural clouds and the variation of cloud droplet concentration. Pure Appl. Geophys., 43, 243-249. 